\title{
Multislice Simulations with Plasmon Energy Losses
}

\section{Budhika Mendis}

\author{
Durham University, Durham, England, United Kingdom
}

The multislice technique is one of the most versatile tools for simulating high energy electron diffraction. It can easily handle crystalline as well as non-crystalline specimens with arbitrary shapes (i.e. the specimen is not restricted to a parallel sided foil). Using the frozen phonon method thermal diffuse scattering can be treated quasi-elastically. However, apart from phonons, the energy loss spectrum is also dominated by other low loss scattering events, notably plasmons. As an example, even in a $10 \mathrm{~nm}$ thick silicon specimen plasmons account for $\sim 10 \%$ of the transmitted intensity, assuming Poisson statistics. In order to accurately model electron-beam specimen interactions it is therefore important to include plasmons within the framework of a frozen phonon simulation.

In this work Monte Carlo methods are used to model plasmon scattering. The Monte Carlo approach treats the incident electron as a particle, and relies on computer generated random numbers to estimate parameters such as the scattering path length and angles. A statistical average of many such scattering events reproduces the key physical parameters (e.g. plasmon mean free path in the case of scattering path length). The concept is similar to frozen phonon, but in addition to phonon 'configurations' we are also using plasmon 'configurations' with variable scattering path length and angles. However, the method only works when electron scattering is particle-like; consequently it is only valid for highly delocalised scattering events such as plasmon excitations. In such cases the periodic nature of the incident electron in the crystal (i.e. Bloch wave) is not important for the scattering cross-section [1].

The plasmon scattering path length and angles follow Poisson and Lorentzian distributions [2], and are governed by the plasmon mean free path $\left(\lambda_{p}\right)$ and characteristic scattering angle $\left(\theta_{E}\right)$ respectively. $\lambda_{p}$ can be measured by recording an EELS spectrum from a specimen of known thickness and comparing the plasmon and zero loss peak intensities. $\theta_{E}$ can be estimated using the formula $\left(E_{p} / 2 E_{\mathrm{o}}\right)$, where $E_{p}$ and $E_{\mathrm{O}}$ are the plasmon and incident electron energies. Alternatively, $\theta_{E}$ can be measured experimentally from the broadening of diffraction spots in an energy filtered selected area diffraction pattern acquired at the plasmon energy. Once $\lambda_{p}$ and $\theta_{E}$ are known the Poisson and Lorentzian distributions can be constructed, and random sampling of these distributions give estimates for the scattering path length and angle respectively. The procedure for including plasmon excitations in frozen phonon simulations is as follows. First the incident electron is propagated through the frozen phonon supercell using the standard procedure for elastic scattering. This is done until the (estimated) scattering depth for plasmon excitation is reached. At this stage the tilt of the incident beam by the plasmon scattering angle must be introduced, i.e. the multislice transmission and propagator functions are modified to that of a tilted beam [2-3]. The electron beam is then propagated through the specimen up to the next plasmon scattering event or until it reaches the specimen exit surface (for the former the transmission and propagator functions must be further updated using the new scattering angle). The signal of interest (e.g. HAADF, diffraction pattern) is obtained by incoherently summing the results from many plasmon configurations.

Figure 1 shows energy filtered $\mathrm{Si}<110>$ diffraction patterns acquired at the zero loss peak as well as single $(17 \mathrm{eV})$, double $(33 \mathrm{eV})$ and triple $(50 \mathrm{eV})$ plasmon energy losses using a $10 \mathrm{eV}$ energy window. The contrast of Kikuchi bands is seen to decrease with increasing energy loss. This trend is also reproduced in the simulated plasmon multislice results (bottom row, Figure 1). Kikuchi bands are formed from Bragg diffraction of the thermal diffuse scattered (TDS) background. High angle TDS scattering requires the electron beam to channel along the atom columns. The channeling is weakened by the small beam 
deflection (of the order of $\theta_{E}$ ) that is present when the electron beam excites a plasmon, which results in lower Kikuchi band contrast. This 'plasmon de-channeling' effect is also responsible for a decrease in the HAADF signal for electrons that have undergone plasmon excitation (Figure 2).

In summary a combined Monte Carlo- frozen phonon multislice method is proposed to include plasmon energy losses in high energy electron diffraction. Plasmon excitations leads to weaker channeling of the electron beam, which is evident from the reduced Kikuchi band contrast observed in energy filtered diffraction patterns [4].

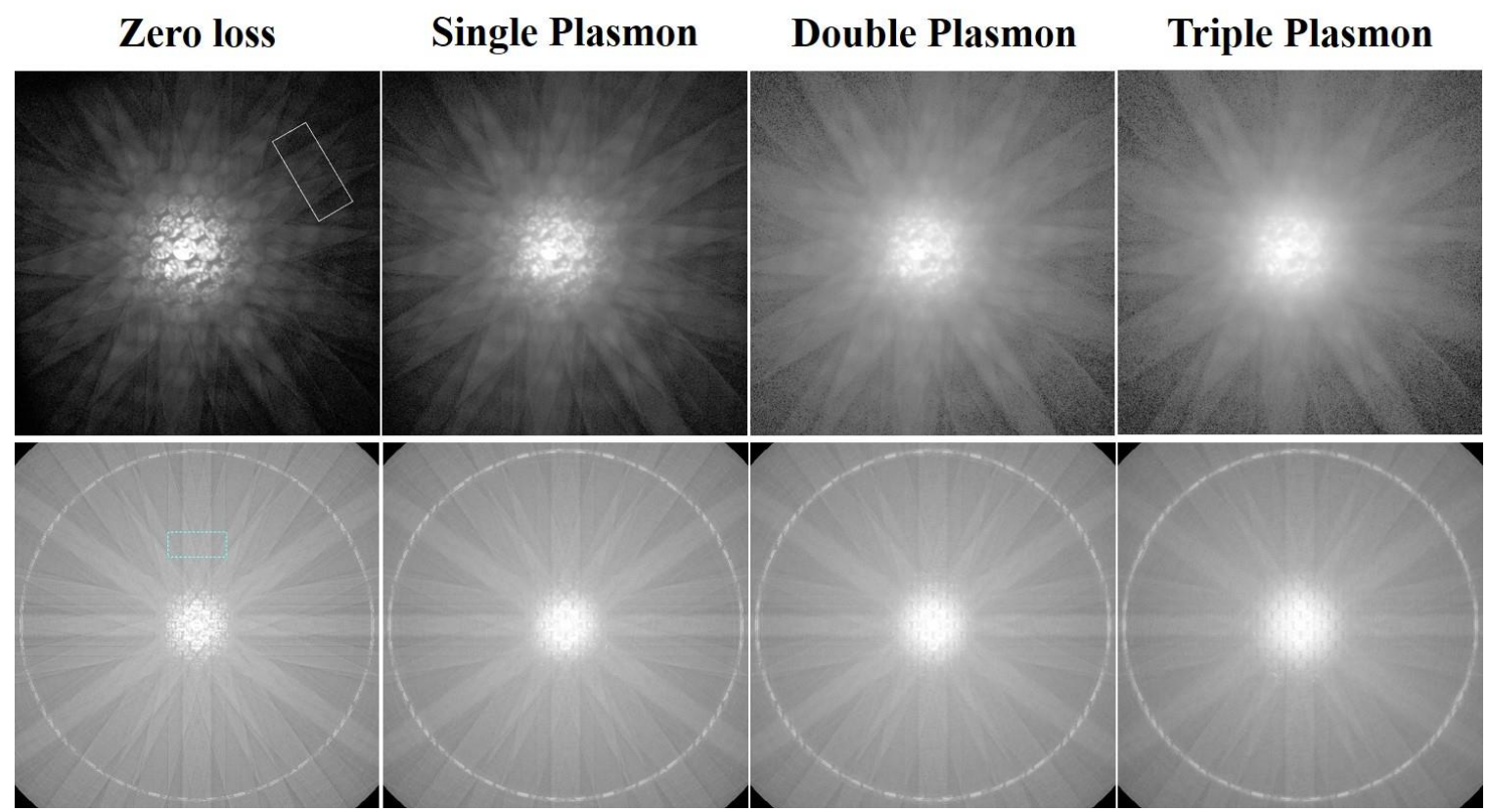

Figure 1. Energy filtered diffraction patterns for a $160 \mathrm{~nm}$ thick, [110] silicon sample acquired at $200 \mathrm{kV}$. Experimental and simulated results are shown in the top and bottom rows respectively.

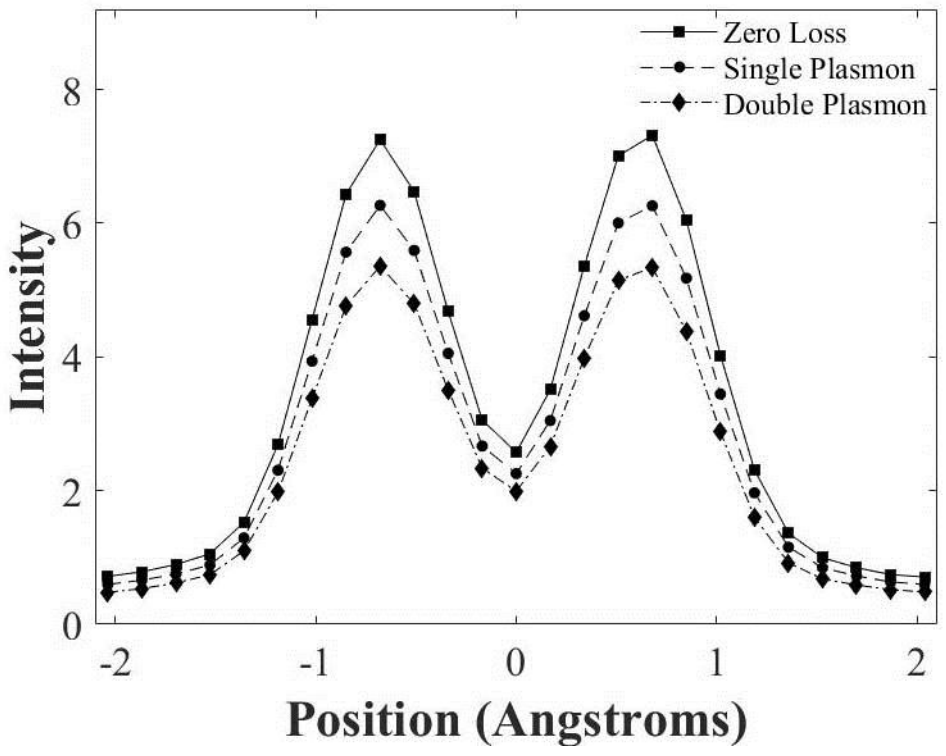

Figure 2. Simulated HAADF intensity profiles across a [110] silicon dumbbell in a $50 \mathrm{~nm}$ thick sample at $200 \mathrm{kV}$. Results are shown for 'zero' loss (including phonons), single and double plasmon energies. 


\section{References}

[1] BG Mendis, Microscopy, DOI: 10.1093/jmicro/dfaa003.

[2] BG Mendis, Ultramicroscopy 206 (2019) 112816.

[3] K Ishizuka, Acta Cryst. A 38 (1982) 773.

[4] BGM would like to thank the North East Centre for Energy Materials (NECEM) for funding (EPSRC grant number EP/R021503/1). 